\title{
Optical and electromagnetic tracking for navigated surgery of the sinuses and frontal skull base*
}

\author{
F. Kral ${ }^{1}$, E.J. Puschban ${ }^{1}$, H. Riechelmann ${ }^{1}$, F. Pedross ${ }^{2}$, W. Freysinger ${ }^{1}$ \\ Department of Otorhinolaryngology, Medical University Innsbruck, Austria \\ 2 Department of Medical Statistics, Informatics and Health Economics, Medical University Innsbruck, Austria
}

SUMMARY

Background: New hardware and software algorithms in electromagnetic tracking for computer assisted surgery (CAS) have been developed. We aimed to compare electromagnetic tracking for navigated procedures in frontal skull base surgery to optical tracking. Methods: Target registration error (TRE) was determined in 6 anatomic specimens in an experimental wet-lab. As targets, 6 titanium screws were evenly distributed over the surgical areas of interest from the frontal sinus to the clivus. Optical tracking and electromagnetic tracking was evaluated in identical software environment using a last generation commercially available navigation system.

Results: Submillimetric application accuracy could be achieved with both tracking modalities. Optical was more accurate than electromagnetic tracking and its reliability was better. Target position did not influence TRE, however TRE varied significantly from skull to skull.

Conclusions: Although less accurate than optical tracking, electromagnetic tracking still offers excellent accuracy and reliability for anterior skull base surgery. Electromagnetic tracking is not dependent on direct line of sight between its hardware components and therefore easily integrated even in cluttered operating theatres.

Key words: navigation, skull base, tracking, CAS

\section{INTRODUCTION}

Computer aided surgery (CAS) is used in sinus and anterior skull base surgery to support the surgeon. In difficult surgical cases, such as revision surgery, when anatomical landmarks are missing, CAS provides positional information of a tracked instrument in preoperatively acquired radiologic imagery. Currently, optical tracking is predominately used for sinus surgery. Active light emitting diodes or passive reflecting spheres are attached to the instruments and the patient for tracking. The position of the patient and the navigated instruments is detected by triangulation of an infrared camera system. To allow detection by the camera system, constant direct line of sight is mandatory. Videoendoscopic sinus surgery e.g. in a four handed transsphenoidal procedure, often results in a cluttered intraoperative setup, where the line of sight for optical navigation systems is not warranted.

Electromagnetic tracking does not need direct line of sight between its components. This technology consists of an emitter to generate a magnetic field and trackers for instruments and the patient, which are located by measuring the electromagnetic field with arrays of coils. The accuracy of electromagnetic tracking was relevantly influenced by ferromagnetic surgical instruments thus leading to unreliable position information intraoperatively ${ }^{(1)}$.

Improvements in hardware and recently developed software algorithms overcome these limitations and accurate ${ }^{(2)}$, reliable results could be achieved in other medical disciplines ${ }^{(3)}$. The new Medtronic S7 navigation system (Medtronic Navigation, Minneapolis, MN, USA) provides software for navigated sinus and skull base surgery either with optical or electromagnetic tracking. The aim of this study was to compare the achievable application accuracy with optical and latest generation electromagnetical tracking in the frontal skull base.

\section{MATERIALS AND METHODS}

The measurements were done in an experimental operating theatre, equipped with standard operating table, lights and instruments. The target registration error (TRE) ${ }^{(4,5)}$ was meas- 
ured in 6 anatomic specimens. IRB approval in experimental setups with anatomic human cadavers is not required by Austrian law and was exempt Austrian law and was exempt from submission to the local ethics committee.

\section{Specimens}

Six formaldehyde-fixed human cadavers were provided by the Department of Anatomy, Medical University Innsbruck. Five anatomic specimens were obtained from female cadavers and 1 from a male cadaver, all aged between 68 to 88 years at the time of death (mean 78.8). Access to the frontal skull base was gained by an axial cut in a supraorbital plane and removal of the brain. Six external fiducial markers (X-Spots, Beekley Corp., Bristol, CT, USA; Ø1.5 mm) were glued to the skin of each specimen for registration and 6 titanium screws were inserted as targets for TRE measurements. The screws were evenly distributed in the posterior wall of the frontal sinus, the ethmoid region and the roof of the sphenoid sinus.

\section{Tracking}

For registration and measurement the Synergy Cranial software (Medtronic, Minneapolis, MN, USA) was used. The software is identical for both tracking modalities which can be selected by the user at the beginning of the workflow. In both settings the dynamic reference frame (DRF) ${ }^{(6)}$ was fixed to the specimen with screws (Framelock system in optical tracking) and a pointer was used for registration and measurement of the titanium screws. The setup is shown in Figure 1.

\section{Imaging}

Each specimen was scanned with a Siemens Somatom 4 row CT (Siemens, Erlangen, Germany) resulting in images with $1 \mathrm{~mm}$ slice thickness $(140 \mathrm{kV}, 220 \mathrm{mAs})$. Image data were transferred from the PACS system to the navigation unit via Ethernet. A surface model was generated automatically by the navigation software by thresholding the data ${ }^{(7)}$.

\section{Registration}

Using the external fiducial markers, a pair-point matching registration was performed ${ }^{(8,9)}$. Identical markers were used for optical and electromagnetical tracking. Two markers were fixed to the forehead, one marker was fixed to each temple, one marker was fixed to the glabella and one marker was fixed to the right lateral wall of the nasal pyramid. After each registration the root mean square (RMS) ${ }^{(10)}$ was displayed as calculated by the software of the navigation system. A high RMS (> $5 \mathrm{~mm}$ ) would not allow the user to switch in the intraoperative navigation modus due to safety reasons. Aside from the choice of the tracking modality, all work steps were identical.

\section{Measurement of TRE}

After each registration TRE was measured at the implanted titanium screws. The tip of the pointer was placed at the center of the Phillips's screw and the deviation of the actual to the displayed position was measured: after placing the probe, the multiplanar view was frozen in maximum magnification (4-fold) and the distances were measured with the navigation system's measurement tool. Deviations were recorded in anterior-posterior (AP), left-right (LR) and head-to-foot (HF) directions. Registrations and measurements were performed by a single person and the setup of each specimen was unaltered from the first registration to the last measurement. Each specimen was measured first with optical and then with electromagnetic tracking.

\section{Repeated measurements}

For each specimen the whole registration and measurement process of the TRE at 6 titanium screws was repeated 8 times per tracking method. Prior to the first registration per specimen, the DRF was mounted for optical tracking and after 8 repetitions of the registration and measurement process, the optical DRF was removed and replaced by the electromagnetic DRF. After that, the registration and measurement was repeated 8 times for electromagnetic tracking. Optical and electromagnetic measurements were performed without interruption in each specimen. The specimen was not moved from the first registration to the last measurement per tracking modality.

\section{Data analysis}

Left-right, anterior-posterior and head-foot deviations were described by means and standard deviations. As a measure of repeatability, the intraclass correlation coefficient of the 8 repetitions of the deviation determinations was calculated using a two-way mixed effects model. A correlation coefficient of 0.2-0.49 was interpreted as low, 0.5-0.69 as moderate, 0.7-0.9 as high and greater than 0.9 as very high. TRE was calculated based on the deviations in space as the length of the resulting euklidian total deviation vector. TRE was described using medians and interquartiles. For further calculations the TRE values of the 8 measurement repetitions were averaged. The Wilcoxon Signed Rank test was used to compare the mean TREs of each tracking modality using the specimen or target as a stratum. The effects of specimen and target were vice versa evaluated with the same test stratified by the tracking modality. Calculations were done with SPSS Ver. 17.0 (Chicago, IL, USA) and StatXact Ver. 8.0 (Cytel Inc, Cambridge, MA, USA). All reported p-values were 2-sided and a type I error level of $5 \%$ was used.

\section{RESULTS}

In total, 1728 deviation measurements resulted from 2 tracking methods, 6 specimens, 6 measurement points and 8 repetitions. The mean deviation in left-right direction was -0.08 ( $\mathrm{sd} 0.28$ ) $\mathrm{mm}$, in anterior-posterior 0.15 (SD 0.4) $\mathrm{mm}$ and in head-tofoot direction 0.46 (SD 0.25$) \mathrm{mm}$. In each direction, a frequency peak at a deviation of $0 \mathrm{~mm}$ could be observed. The total mean TRE was $0.28 \mathrm{~mm}$ with a SD of $0.5 \mathrm{~mm}$ and a maximum of $3.12 \mathrm{~mm}$. The TRE distribution was not normal. 
Table 1. Target registration error (TRE) at six implanted titanium screws in the frontal skull base (both registration modalities). Target position did not influence TRE $(\mathrm{p}=0.96)$.

\begin{tabular}{|c|c|c|c|c|}
\hline & \multicolumn{3}{|c|}{ TRE in $\mathrm{mm}$} \\
\hline & & Median & $\begin{array}{l}\text { Percentile } \\
25\end{array}$ & $\begin{array}{l}\text { Percentile } \\
\quad 75\end{array}$ \\
\hline \multirow{6}{*}{ 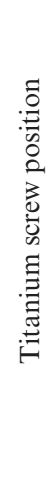 } & $\begin{array}{l}\text { Posterior wall of left } \\
\text { frontal sinus }\end{array}$ & 0.18 & 0.03 & 0.33 \\
\hline & $\begin{array}{l}\text { Posterior wall of right } \\
\text { frontal sinus }\end{array}$ & 0.24 & 0.07 & 0.46 \\
\hline & $\begin{array}{l}\text { Left posterior ethmoid } \\
\text { foramen }\end{array}$ & 0.28 & 0.04 & 0.57 \\
\hline & $\begin{array}{l}\text { Right posterior eth- } \\
\text { moid foramen }\end{array}$ & 0.32 & 0.15 & 0.39 \\
\hline & $\begin{array}{l}\text { Roof of left sphenoid } \\
\text { sinus }\end{array}$ & 0.31 & 0.13 & 0.42 \\
\hline & Clivus & 0.15 & 0.08 & 0.54 \\
\hline
\end{tabular}

Table 2. Target registration error (TRE) as a measure of accuracy for optical and electromagnetic tracking in the frontal skull base. Optical tracking was significantly more accurate than electromagnetic tracking $(\mathrm{p}<0.001)$.

\begin{tabular}{|c|c|c|c|c|}
\hline & & \multicolumn{3}{|c|}{ TRE in $\mathrm{mm}$} \\
\hline & & Median & $\begin{array}{c}\text { lower } \\
\text { quartile }\end{array}$ & $\begin{array}{r}\text { upper } \\
\text { quartile }\end{array}$ \\
\hline \multirow{2}{*}{ 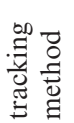 } & optical & 0.12 & 0 & 0.32 \\
\hline & electro-magnetic & 0.37 & 0.18 & 0.53 \\
\hline
\end{tabular}

\section{Specimen and target position}

The specimens had a significant effect on TRE ( $p=0.001$, Figure 2). Depending on the anatomical specimen used, TREs ranged from median $0.03 \mathrm{~mm}(0 \mathrm{~mm}$ to $0.19 \mathrm{~mm}$, lower and upper quartiles) to $0.43 \mathrm{~mm}(0.3 \mathrm{~mm}$ to $0.66 \mathrm{~mm})$. The target position did not affect TRE ( $p=0.94$, Table 1$)$. The median was between 0.15 and $0.32 \mathrm{~mm}$, the lower quartiles ranged from 0.03 to $0.15 \mathrm{~mm}$ and the upper quartiles from 0.33 to 0.57 $\mathrm{mm}$. There was no relevant difference between target points near the surface or near the center of the head.

\section{Tracking}

The effect of tracking modality on TRE was determined by averaging the results of the 8 repetitions. Six targets and 6 specimens resulted in 36 electromagnetic and 36 optical tracking measurements. The choice of tracking modality had a significant influence on TRE $(p<0.001)$. The median TRE for electromagnetic tracking was 0.37 ( 0.18 to 0.53$) \mathrm{mm}$ and for

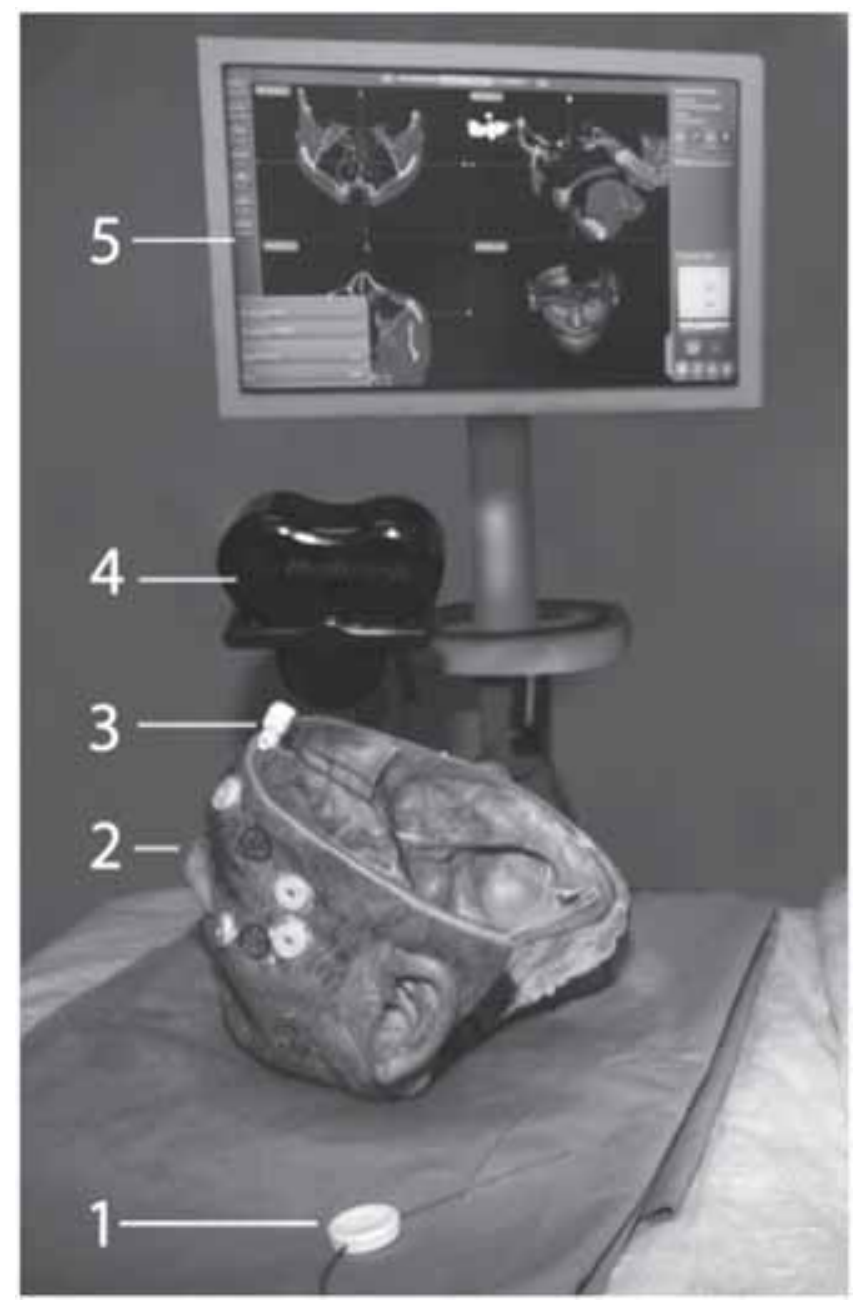

Figure 1. Wet-lab setup with electromagnetic tracking: pointer (1), anatomic specimen (2), screw mounted dynamic reference element (3), electromagnetic field emitter (4) and the surgeon's monitor (5) to visualize the position of the instrument's tip in the multiplanar reconstruction of the preoperative acquired image data.

optical tracking was 0.12 (0.0 to 0.32$) \mathrm{mm}$ (median, lower and upper quartiles, respectively; Table 2).

\section{Repeatability}

The intraclass correlation coefficients of the 8 repetitions of LR, AP and HF measurements were determined to assess the repeatability of the whole registration process (Figure 3). For LR measurements the intraclass correlation coefficient was $0.61(95 \% \mathrm{CI}=0.46$ to $0.73, \mathrm{p}<0.001)$, for AP measurements it was $0.56(95 \% \mathrm{CI}=0.39$ to $0.7, \mathrm{p}<0.001)$, and for HF measurements it was $0.64(95 \% \mathrm{CI}=0.46$ to $0.75, \mathrm{p}<0.001)$.

\section{DISCUSSION}

Optical tracking has established itself as a de facto standard in intraoperative navigation ${ }^{(11)}$. It provides submillimetric spatial resolution ${ }^{(12,13)}$, but often fails due to line of sight problems in cluttered operating theatres. Electromagnetic tracking is an alternative technology to optical tracking. Magnetic tracking 


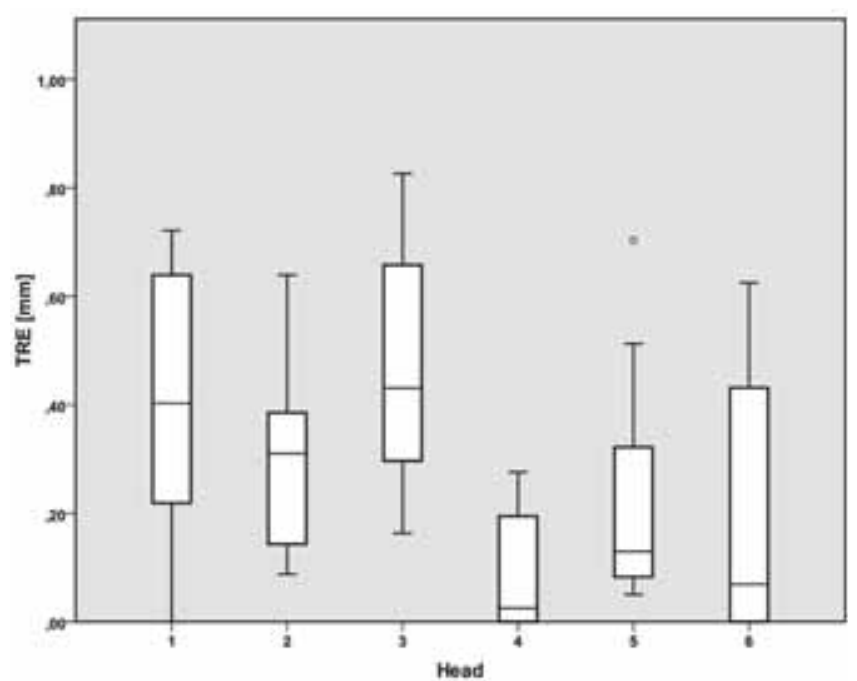

Figure 2. Box-plot of target registration error (TRE) of 6 titanium screw targets in 6 human cadaver heads after electromagnetic or optical registration (each box represents medians, upper and lower quartiles of 12 averaged measurements). TRE differed significantly from head to head $(\mathrm{p}=0.001)$

technology suffers from its inherent sensitivity to the presence of magnetisable material in the operating field ${ }^{(14,15)}$. Recent developments paved the way for reliable application of magnetic position sensing technology ${ }^{(16,17)}$. Last generation hardware and new software algorithms are reported to be more accurate and more resistant to ferromagnetic distortions allowing surgical use ${ }^{(18,19)}$. The Medtronic S7 navigation system offers both tracking modalities in an identical software environment and thus allows for direct comparison of optical and electromagnetic tracking for navigated endoscopic procedures.

This study was to determine whether accuracy of electromagnetic tracking is comparable to that of optical tracking for frontal skull base surgery. Standard surgical equipment was used in an experimental wet-lab in order to simulate intraoperative findings as closely as possible. TRE was calculated and served as a measure of clinically achievable application accuracy ${ }^{(20,21)}$

In this study, optical tracking was significantly more accurate than electromagnetic tracking. However, sub-millimetric application accuracy was achieved with both modalities under experimental wet-lab conditions. This suggests that clinical application accuracy at or below the $1 \mathrm{~mm}$ range will be possible also with electromagnetic tracking.

Titanium screws served as target points to measure TRE. The titanium screws were implanted next to surgical areas of interest. Positioning the tip of the targeting probe in the head of a titanium screw results in high accuracy. Surprisingly, the repeatability of TRE measurements in the titanium screws was only moderate (Figure 3), particularly in electromagnetic tracking. A possible reason for this is high variability in the

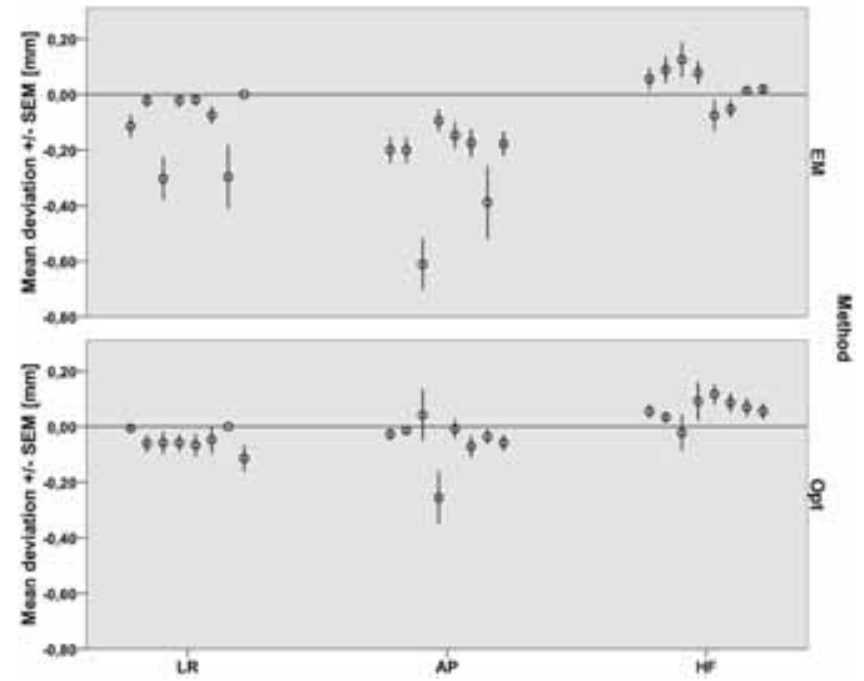

Figure 3. Mean \pm standard error of mean (SEM) of 8 repetitions of 36 deviation measurements in left-right (LR), anterior-posterior (AP) and head-foot (HF) direction, grouped by either electromagnetic (EM) or optical (Opt) tracking. Larger deviations from the zero-line mean less accuracy, higher data variability means less reliability.

registration process as recently reported ${ }^{(22)}$. In respect of this matter, all specimens were measured by one ENT-surgeon with long-standing experience in CAS. A DRF was screwed directly to the specimen's bone to avoid any bias when the specimen accidentally would have been moved. Each specimen was measured in one session to eliminate alterations in the experimental setup.

The results of this study were obtained in anatomical specimens from an experimental setup. It is understood that the results cannot be transferred to a clinical setup one to one. However, both tracking methods are equally affected by differences between real world conditions and the setup in a wet-lab, where hundreds of measurements were averaged. In conclusion, electromagnetic tracking in CAS interventions of the frontal skull base offered submillimetric, reliable application accuracy. Electromagnetic tracking is easily integrated in daily clinical workflow and superior to optical tracking in cluttered operating theatres. This advantage however, comes for the price of a slightly lower application accuracy.

\section{ACKNOWLEDGEMENTS}

The authors would like to thank Romed Hörmann, Department of Anatomy, Medical University Innsbruck for his support in preparing the anatomical specimens and Peter Bauer for photo editing.

\section{AUTHOR CONTRIBUTIONS}

FK: Study design, writing, interpretation, data acquisition, approval. EJP: Data acquisition, data analysis, editing, approval. HR: Statistics, interpretation, writing, editing, approval. FP: statistics, interpretation, drafting, approval. WF: Study design, interpretation, drafting, writing, approval. 


\section{CONFLICT OF INTEREST STATEMENT}

The authors do not have any financial or personal conflict of interests. This study was carried out in an academic setting without any financial support by companies.

\section{REFERENCES}

1. Birkfellner W, Watzinger F, Wanschitz F, Enislidis G, Kollmann C, Rafolt D, et al. Systematic distortions in magnetic position digitizers. Med Phys. 1998; 25: 2242-2248.

2. Schicho K, Figl M, Donat M, et al. Stability of miniature electromagnetic tracking systems. Phys Med Biol. 2005; 50: 2089-2098.

3. Graydon AJ, Malak S, Anderson IA, Pitto RP. Evaluation of accuracy of an electromagnetic computer-assisted navigation system in total knee arthroplasty. Int Orthop. 2009; 33: 975-979.

4. Fitzpatrick JM, West JB, Maurer CR, Jr. Predicting error in rigidbody point-based registration. IEEE Trans Med Imaging. 1998;17: 694-702.

5. Labadie RF, Davis BM, Fitzpatrick JM. Image-guided surgery: what is the accuracy? Curr Opin Otolaryngol Head Neck Surg. 2005; $13: 27-31$.

6. Zheng G, Caversaccio M, Bachler R, Langlotz F, Nolte LP, Hausler R. Frameless optical computer-aided tracking of a microscope for otorhinology and skull base surgery. Arch Otolaryngol Head Neck Surg. 2001; 127: 1233-1238.

7. Udupa JK, Herman GT. 3D Imaging in Medicine. 2 ed. Boca Raton, FL, USA: CRC Press; 2000.

8. Fitzpatrick JM, West JB. The distribution of target registration error in rigid-body point-based registration. IEEE Trans Med Imaging. 2001; 20: 917-927.

9. Knott PD, Batra PS, Butler RS, Citardi MJ. Contour and paired-point registration in a model for image-guided surgery. Laryngoscope. 2006; 116: 1877-1881.

10. West JB, Fitzpatrick JM, Toms SA, Maurer CR, Jr., Maciunas RJ. Fiducial point placement and the accuracy of point-based, rigid body registration. Neurosurg. 2001; 48: 810-816.

11. Caversaccio M, Freysinger W. Computer assistance for intraoperative navigation in ENT surgery. Minim Invasive Ther Allied Technol. 2003; 12: 36-51.

12. Li Q, Zamorano L, Jiang Z, et al. Effect of Optical Digitizer Selection on the Application Accuracy of a Surgical Localization System-A Quantitative Comparison between the OPTOTRAK and FlashPoint Tracking Systems. Comput Aided Surg. 1999; 4: 314-321.

13. Chassat F, Lavallée S. Experimental Protocol of Accuracy Evaluation of 6-D Localizers for Computer-Integrated Surgery: Application to Four Optical Localizers. Lecture Notes in
Computer Science; Berlin, Heidelberg: Springer-Verlag; 1998 p. 277-284.

14. Milne AD, Chess DG, Johnson JA, King GJ. Accuracy of an electromagnetic tracking device: a study of the optimal range and metal interference. J Biomech. 1996; 29: 791-793.

15. Reinhardt H, Trippel M, Westermann B, Gratzl O. Computer aided surgery with special focus on neuronavigation. Comput Med Imaging Graph. 1999; 23: 237-244.

16. Frantz DD, Wiles AD, Leis SE, Kirsch SR. Accuracy assessment protocols for electromagnetic tracking systems. Phys Med Biol. 2003; 48: 2241-2251

17. Santanam L, Noel C, Willoughby TR, et al. Quality assurance for clinical implementation of an electromagnetic tracking system. Med Phys. 2009; 36: 3477-3486.

18. Hummel J, Figl M, Birkfellner W, et al. Evaluation of a new electromagnetic tracking system using a standardized assessment protocol. Phys Med Biol. 2006; 51: N205-N210.

19. Yaniv Z, Wilson E, Lindisch D, Cleary K. Electromagnetic tracking in the clinical environment. Med Phys. 2009; 36: 876-892.

20. Hardy SM, Melroy C, White DR, Dubin M, Senior B. A comparison of computer-aided surgery registration methods for endoscopic sinus surgery. Am J Rhinol. 2006; 20: 48-52.

21. Labadie RF, Shah RJ, Harris SS, et al. In vitro assessment of image-guided otologic surgery: submillimeter accuracy within the region of the temporal bone. Otolaryngol Head Neck Surg. 2005; 132: 435-442.

22. Kral F, Riechelmann H, Freysinger W. Navigated surgery at the lateral skull base is strongly influenced by registration and preoperative imagery. Laboratory results. Arch Otolaryngol Head Neck Surg. 2011; 137: 144-150.

Florian Kral, MD

Department of Otorhinolaryngology

Medical University Innsbruck

Anichstraße 35

6020 Innsbruck

Austria

Tel: $+43-512-50481679$

Fax: $+43-512-50423140$

E-mail: florian.kral@i-med.ac.at 